\title{
Evolution of thorax architecture in ant castes highlights trade-off between flight and ground behaviors
}

\author{
Roberto A Keller ${ }^{1,2 *}$, Christian Peeters ${ }^{2}$, Patrícia Beldade ${ }^{1}$ \\ ${ }^{1}$ Instituto Gulbenkian de Ciência, Oeiras, Portugal; '2Laboratoire Écologie \& Évolution, \\ CNRS UMR 7625, Université Pierre et Marie Curie, Paris, France
}

\begin{abstract}
The concerted evolution of morphological and behavioral specializations has compelling examples in ant castes. Unique to ants is a marked divergence between winged queens and wingless workers, but morphological specializations for behaviors on the ground have been overlooked. We analyzed thorax morphology of queens and workers in species from 21 of the 25 ant subfamilies. We uncovered unique skeletomuscular modifications in workers that presumably increase power and flexibility of head-thorax articulation, emphasizing that workers are not simply wingless versions of queens. We also identified two distinct types of queens and showed repeated evolutionary associations with strategies of colony foundation. Solitary founding queens that hunt have a more worker-like thorax. Our results reveal that ants invest in the relative size of thorax segments according to their tasks. Versatility of head movements allows for better manipulation of food and objects, which arguably contributed to the ants' ecological and evolutionary success.
\end{abstract}

DOI: 10.7554/eLife.01539.001

*For correspondence: roberto. kellerperez@gmail.com

Competing interests: The authors declare that no competing interests exist.

Funding: See page 16

Received: 13 September 2013 Accepted: 27 November 2013 Published: 07 January 2014

Reviewing editor: Diethard Tautz, Max Planck Institute for Evolutionary Biology, Germany

(c) Copyright Keller et al. This article is distributed under the terms of the Creative Commons Attribution License, which permits unrestricted use and redistribution provided that the original author and source are credited.

\section{Introduction}

A detailed understanding of morphology is of prime importance to elucidate how organisms evolved and operate in nature. This is especially so in an era of increasingly sophisticated developmental genetic analysis, as the correct interpretation of molecular data depends largely on the precise characterization of morphological structures (e.g., Prud'homme et al., 2011 vs Yoshizawa, 2011; Mikó et al., 2012). During development, the relative investment in the growth of different body parts (e.g., allocation of nutritional resources to somatic vs germ tissues) will determine adult morphologies, and thus influence an organism's ecology (Nijhout and Emlen, 1998; Emlen, 2001). Morphology interacts very closely with behavior in shaping phenotypic evolution (Baldwin, 1896; Simpson, 1953; Robinson and Dukas, 1999). On the one hand, changes in behavior will often influence the environment in which organisms are selected, leading to modifications of morphology (Wcislo, 1989; Crispo, 2007). On the other hand, morphological specializations can open the potential for further behavioral change (West-Eberhard, 2003).

Specializations that associate morphology and behavior have compelling examples in insect polyphenisms, where alternative morphologies result from environmental regulation of development and are typically associated with distinct behavioral repertoires (Beldade et al., 2011; Simpson et al., 2012). For example, horned and hornless male beetles produced as a result of nutritional plasticity have different reproductive tactics (guarding vs sneaking access to females in nests [Moczek and Emlen, 2000]). In many social insects, differential feeding leads to the production of distinct queen and worker castes, each with characteristic morphology and behavior underlying reproductive vs maintenance functions within the colony (Wheeler, 1986; Beldade et al., 2011), and increasing colony performance as a whole (Oster and Wilson, 1978).

Among the social Hymenoptera, ants are an extreme case of caste polyphenism, because queens are usually winged and workers are always wingless (Wilson, 1971; Hölldobler and Wilson, 1990). Flight allows queens to disperse from the natal nests before they start new colonies, while the lack of 
eLife digest The size and shape of an animal, known as its morphology, often reflect the actions it can perform. A grasshopper's long legs, for example, are well suited to hopping, whilst the streamlined body of a dolphin helps swimming through water. These specialized features result from the interplay between morphology and behavior during evolution. A change in morphology can make new behaviors possible, which can then expose the animal to new environments and selective pressures that, in turn, can lead to further changes in morphology.

The interplay between morphology and behavior is particularly interesting in social insects such as ants. Queens and workers within an ant colony have a similar set of genes, but they have dramatically different morphologies and very different roles within the colony. Queens are responsible for reproduction, and are larger and have wings, which allow them to fly and establish a new colony away from where they were born. Workers are smaller and lack wings, and they devote themselves to building the nest, feeding the young larvae and protecting the colony. This marked morphological divergence, unique to ants, has fascinated researchers for more than a century. However, most studies have focused on the presence or absence of wings and have overlooked the interactions between morphology and the actions performed on the ground.

Like all insects, an ant's body is divided into three parts: the head, the thorax (to which the legs and wings are attached), and the abdomen. Now, Keller et al. have examined the shape of the thorax in many species of ants and found that workers are not just smaller wingless versions of queens: rather, the architecture of their thorax is unique among species of flying insects. The front end of the worker thorax is greatly enlarged and is filled by strong neck muscles that power the head and its jaws, and allow workers to hunt and carry prey many times their own weight.

Keller et al. also identified two distinct types of queens and went on to show that these two shapes evolved in association with the two types of strategy that lone queens use to found new colonies. In species where queens convert their own wing muscles into the food for the first generation of workers, the wing muscles are much enlarged and the neck segment is extremely reduced. In species where queens hunt to feed the new colony, the wing and neck muscles are more balanced in size. As such, for those ant species where very little is known about how new colonies are founded, Keller et al. show that we can use the shape of the queen's thorax to help predict this behavior.

Taken together, the results of Keller et al. show that female ants invest in the relative size of the different segments of the thorax in a way that reflects their behavior as adults. These adaptations partly explain why ants have been so extraordinarily successful in nature, and underscore the importance of carefully analyzing an organism's form to fully understand its biology.

DOI: 10.7554/eLife.01539.002

wings in workers is thought to facilitate the exploitation of ground habitats and cramped spaces (Hölldobler and Wilson, 1990). The presence and operation of wings is tightly associated with the morphology of the thorax. In the typical thorax of modern flying insects, the first segment (T1) bears no dorsal appendages, while the second (T2) and third (T3) each bear a pair of wings (Snodgrass, 1935). Because of this, studies of morphological specializations of the insect thorax have focused on the wing-bearing segments $T 2$ and $T 3$. The relative size of these segments varies widely across insect orders, but tends to be conserved within (Dudley, 2002). Surprisingly, the entire thoracic skeletomuscular architecture of ant castes, including the T1 segment that forms the articulation with the head, has been neglected, from both functional and comparative perspectives.

In this study, we use a phylogenetically broad comparative approach, involving queens and workers from species representing 21 of the 25 extinct and extant ant subfamilies, to investigate external morphology and internal anatomy in the context of caste-specific specialized behaviors. Our analysis reveals a unique modification of the thoracic architecture in worker ants, presumably connected with their powerful head and mandibles, and uncovers two types of thoracic configurations in queens, associated to different strategies for the foundation of new colonies.

\section{Results}

To characterize caste-specific modifications in the architecture of the thorax, we first quantified the length of the thoracic segments in queens and workers of 11 species and performed anatomical 
dissections in multiple individuals from 19 species, representing eight ant subfamilies. We unveiled a unique thorax architecture in workers vs queens and then confirmed the generality of our findings in an extended sample of species across ant diversity. The quantitative analysis of thorax morphology showed that queens fall in two distinct anatomical types. Using parsimony and maximum likelihood (ML) methods, we reconstructed the pattern of thoracic evolution onto the established phylogenetic tree of the ants (Brady et al., 2006; Moreau et al., 2006) for 54 genera representing 21 subfamilies plus two genera of wasps as outgroups. We also compiled behavioral data on the mode of colony foundation for our exemplar species and tested for correlated evolution between queen thoracic morphology and founding behavior. Our comparisons are drawn from a total of 111 species belonging to 93 genera within Formicidae, representing 20 of the 21 extant subfamilies plus the fossil taxon Sphecomyrminae ${ }^{\dagger}$ among the four extinct subfamilies.

\section{The unique thoracic architecture of worker ants}

We assessed the relative sizes and configuration of the dorsal plates that form the thoracic exoskeleton in 265 queens and workers belonging to 11 species in five major ant subfamilies (Table 1). For each caste of each species, we measured the length of $\mathrm{T} 1, \mathrm{~T} 2$, and $\mathrm{T} 3$ of $5-17$ individuals from museum collections. Our morphometric analyses showed that in ant queens, both T1 and T3 are reduced relative to T2, which makes up most of the thorax (Figure 1A). This conforms to the typical proportions in insects where flight is powered exclusively by large wing muscles inside T2 (Snodgrass, 1935; Dudley, 2002) (e.g., Diptera, Hymenoptera, and Lepidoptera). In contrast, in ant workers, T1 is greatly enlarged and forms a significant portion of the thorax, while T2 is reduced (illustrative SEM image in Figure 2). $\mathrm{T} 3$ is absent dorsally in workers of most species but, when T3 is distinguishable, the T3/T2 ratio does not differ between castes. In contrast, the ratio between T1 and T2 clearly discriminates workers and

Table 1. Ant species studied for morphometrics and/or internal anatomy

\begin{tabular}{llcccr} 
& & Morphometrics & Dissections \\
\hline Subfamily & Species & $\mathbf{q}$ & w & q & w \\
\hline Amblyoponinae & Amblyopone australis & 6 & 8 & 2 & 2 \\
\hline Dolichoderinae & Tapinoma simrothi & - & - & 6 & 10 \\
\hline Ectatomminae & Ectatomma ruidum & - & - & 3 & 5 \\
\hline Formicidae & Lasius niger & 15 & 15 & 2 & 4 \\
\hline & Oecophylla smaragdina & - & - & 2 & 5 \\
\hline Myrmeciinae & Polyrhachis laboriosa & 13 & 5 & 3 & 8 \\
\hline & Myrmecia simillima & - & - & 2 & 4 \\
\hline Myrmicinae & Nothomyrmecia macrops & - & - & 1 & 4 \\
\hline & Carebara vidua & 5 & 3 & 1 & 1 \\
\hline & Cataulacus wasmanni & 15 & 15 & 3 & 3 \\
\hline & Leptothorax pergandei & 13 & 15 & 1 & 3 \\
\hline Messor barbarus & - & - & 3 & 8 \\
\hline Ponerinae & Monomorium pharaonis & 15 & 15 & 2 & 4 \\
\hline & Monomorium subopacum & - & - & 2 & 3 \\
\hline & Pogonomyrmex barbatus & 15 & 17 & 4 & 5 \\
\hline & Brachyponera lutea & 15 & 15 & 3 & 5 \\
\hline & Harpegnathos saltator & - & - & 2 & 4 \\
\hline Neoponera apicalis & 7 & 12 & 4 & 10 \\
\hline & Tetraponera aethiops & 11 & 15 & 4 & 6 \\
\hline
\end{tabular}

$q=$ number of queens examined; $w$ = number of workers examined. Generic placement of Brachyponera lutea and Neoponera apicalis reflects the new reclassification of species within the former paraphyletic genus Pachycondyla (Schmidt CA, Shattuck SO, The higher classification of the ant subfamily Ponerinae [Hymenoptera: Formicidae], with a review of ponerine ecology and behavior. Under review)

DOI: 10.7554/eLife.01539.003 


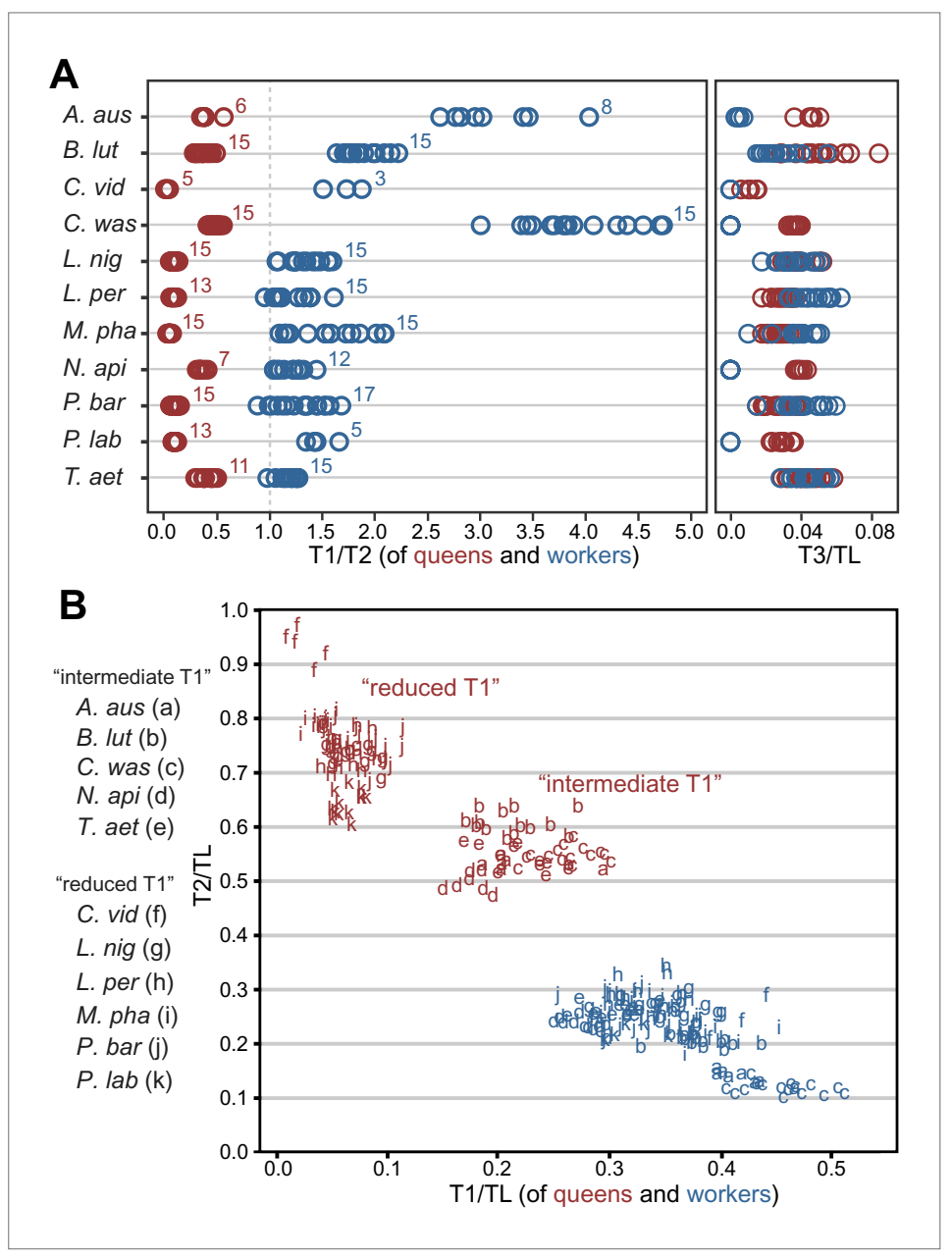

Figure 1. Variation in length of first (T1) and second (T2) thoracic segments in ants shows characteristic differences depending on caste and species. (A) Relative lengths of T1 and T2 (left) show clear differences between queens and workers for 11 ant species. T3 (right) constitutes a small portion of the total length of the thorax in both queens and workers and, when present (when T3/TL > 0.0), is indistinguishable between castes. Numbers correspond to sample sizes and are equal for both panels (Table 1). (B) Gradient of investment in neck strength vs flight/storage musculature sorts individuals into three categories. Queens fall into two discrete categories based on the relative lengths of $\mathrm{T} 1$ and $\mathrm{T} 2$. While the use of T1/T2 in (A) emphasizes the distinction between workers and queens and within species variation, $\mathrm{T} 1 / \mathrm{TL}$ and $\mathrm{T} 2 / \mathrm{TL}$ in (B) enables the distinction between queen types across species with large differences in body size. Measurements and ratios are available in the Dryad data repository under DOI doi: 10.5061/dryad.d62p2/1 (Keller et al., 2014). Species codes: A. aus = Amblyopone australis; B. lut = Brachyponera lutea; C. vid = Carebara vidua; C. was = Cataulacus wasmanni; L. nig $=$ Lasius niger; L. per $=$ Leptothorax pergandei; M. pha $=$ Monomorium pharaonis; $N$. api $=$ Neoponera apicalis; $P$. bar $=$ Pogonomyrmex barbatus; $P$. lab $=$ Polyrhachis laboriosa; $T$. aet $=$ Tetraponera aethiops.

DOI: 10.7554/eLife.01539.004

The following figure supplements are available for figure 1:

Figure supplement 1. Measurements used in this study. DOI: 10.7554/eLife.01539.005

Figure supplement 2. Differences in length proportion of thoracic segments among castes in nine representative species from different subfamilies.

DOI: 10.7554/eLife.01539.006

queens. Rather than just showing an overall reduction in T2, consistent with their lack of wings, worker ants have a T1/T2 ratio reversed in relation to queens (Figure 1A; SEM images in Figure 2). The difference between castes in this ratio depends on the species (Linear model: interaction Species $\mathrm{x}$ Caste, $\mathrm{df}=10, \mathrm{~F}=68.3, \mathrm{p}<0.00001$ ) but it is always greater in workers than in queens (Linear Model: holding the factor Species constant, factor Caste, $d f=1, F=8975.3, p<0.00001)$. Visual inspection of 


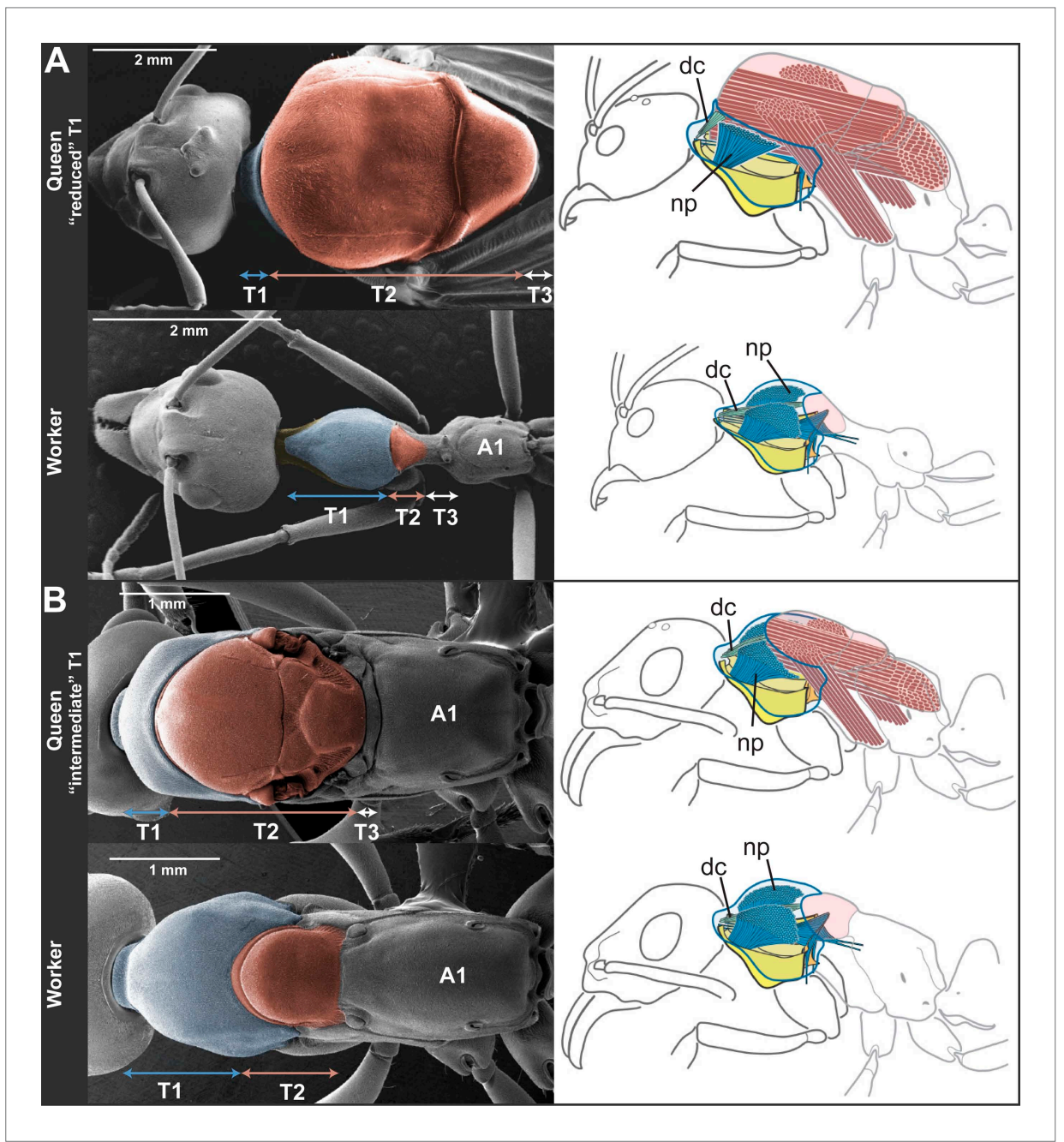

Figure 2. Skeletomuscular specialization of queens and workers in ants. The dorsal plate of T1 is always enlarged in workers relative to queens (left column; multiple individuals from 52 genera examined, Table 2). Queens can either (A) have a reduced T1 and huge T2-associated wing muscles (represented here by Oecophylla smaragdina), or (B) show a slightly enlarged T1 and associated neck muscles (represented here by Neoponera apicalis). T1, T2, and T3, first, second and third thoracic segments respectively; A1, first abdominal segment. Workers of N. apicalis lack a discernible T3. Internally (right column), the wing muscles in queens (red) fill most of the thoracic cavity, while the T1 muscles (blue) are narrow and close to the thoracic wall. In all workers examined (see Table 1 for list of species and sample sizes), the T1 notopleural muscles (np, dark blue) that support the anteroventral plates (yellow) fill the anterior portion of the cavity. The dorsal cervical muscles ( $d c$, light blue; see also Figure 2-figure supplement 1B) that in winged queens originate at the anterior phragma and pull the head up at contraction, show a shifted position in workers. In the absence of phragma, these muscles originate at the dorsal boundary between T1 and T2. Rather than being short and thin, they form long and thick bundles that stretch the entire length of the enlarged T1 cavity to their place of insertion on the back of the head (Figure 2-figure supplement 2). Figure 1-supplement 1 has photos of many more species of 'reduced T1' and 'intermediate T1' species for comparison of external thoracic morphology.

DOI: 10.7554/eLife.01539.007

The following figure supplements are available for figure 2:

Figure supplement 1. Thoracic musculature in queen and worker ants.

DOI: 10.7554/eLife.01539.008

Figure supplement 2. Internal anatomical adaptations in ant workers for powerful head movement.

DOI: 10.7554/eLife.01539.009 
an extended sample of species (Table 2) from 21 of the 25 ant subfamilies (including the extinct taxon Sphecomyrminae ${ }^{\dagger}$ ) confirmed the universality of these relative length differences. Castes of all species with specimens available show a differential investment in the growth of T1 and T2. T2 was larger than T1 in queens of all 52 species examined, and T1 was larger than T2 in workers of all 111 species examined (Table 2; examples in Figure 1-figure supplement 2).

To infer the functional significance of the caste-specific external thoracic configurations, we performed a comparative analysis of the internal skeletomuscular system in queens and workers. We dissected 144 individuals from 19 species belonging to eight subfamilies (Table 1) and analyzed both muscle (extent of attachment) and skeletal elements. Our dissections showed that the length of the thoracic segments in dorsal view is a reflection of the volume of the muscles associated with each segment. In the same way that the large T2 of queens is indicative of the presence of large wing muscles, the large T1 in workers reflects the enlargement of muscles in this segment (Figure 2, Figure 2-figure supplement 1, Figure 2-figure supplement 2). Studies in other insects established that homologous T1-associated skeletomuscular elements are involved in the head-thorax articulation or neck (Snodgrass, 1935, 1956; Hartenstein, 2006). In queens of all 19 ant species dissected (Table 1), the neck-associated muscles were short and thin, traversing the narrow space of T1 between the head and the anterior phragma (cuticular invagination) of T2 where the wing muscles attach (Figure 2, Figure 2-figure supplement 1A). This configuration of neck elements is similar to that of female honey bees irrespective of caste (Snodgrass, 1956), and Drosophila fruit flies (Hartenstein, 2006; McQuilton et al., 2012). In contrast, in ant workers, the expansion of T1 and the lack of both anterior phragma and wing muscles result in a larger anterior cavity that contains neck muscles and skeletal pieces in a unique configuration.

The most striking muscular difference between ant castes concerns one of the notopleural pairs of muscles that originate dorsally on T1 (np in Figure 2, right column; Figure 2-figure supplement 1C-H). The main function of homologous muscles in honey bees is to carry the plates that support the head and serve to move it sideways or rotate it (Snodgrass, 1956). In ant queens, where most of the thoracic cavity is filled by the wing muscles (as is the case in all castes of honey bees), these muscles are narrow and close to the thoracic wall. Our dissections revealed that the equivalent muscles in ant workers are hypertrophied, and fill the wider T1 cavity completely. Ant workers also show important differences in internal skeleton associated with T1 (Figure 2-figure supplement 2B). This skeletomuscular configuration highlights the increased strength of the workers' neck that powers head movements.

\section{Two types of queen thoracic architecture}

Even though queens invest mostly in the thoracic segment used for flight (T2), our morphometric data showed that queens of different species fall into two discrete categories based on the relative investment into T1. When plotting the normalized length of T1 vs T2 for 130 queens measured (Table 1), we can discriminate two clusters of species (Figure 1B; where workers of all species form a third cluster). For five of the 11 species in Table 1, queens have a reduced T1, almost not visible in dorsal view (Figure 2A). The other six species form a category with queens having an intermediate T1, corresponding to enlarged T1 muscles (Figure 2B).

To investigate the evolution of queen thoracic configurations across the ant phylogeny, we focused on a total of 54 ant species (those in Table 2 for which queens were available for measurements) representing 21 subfamilies, as well as two species of wasps from different families as outgroups (Table 3). Queens were scored as belonging to the categories 'reduced T1' (22 species, all with T1/T2 $<0.14$ ) or 'intermediate T1' (32 species, all with T1/T2 > 0.28), as seen in Figure 1B. This information was combined with a well-established ant phylogeny (Brady et al., 2006; Moreau et al., 2006) and we used parsimony and maximum likelihood (ML) methods to reconstruct ancestral character states (Figure 3). Our analysis showed that an 'intermediate T1' in queens arose in the common ancestor to all ants (ML proportional likelihood $=0.800)$, and that there were multiple transitions to the 'reduced T1' (Figure 3). This reduction seems to have evolved convergently in at least four major ant lineages. Transitions back to an 'intermediate T1' are rare and more recent events, being restricted to the genera Polyergus within subfamily Formicinae, and Cataulacus and Metapone within subfamily Myrmicinae. In contrast, the universal occurrence of a hypertrophied T1 in workers (including the primitive fossil Sphecomyrma ${ }^{\dagger}$ ) supports a single origin of this novel thoracic configuration in the common ancestor of all ants (Figure 3). 
Table 2. List of species surveyed for relative length of thoracic segments

\begin{tabular}{|c|c|c|c|c|c|}
\hline \multirow[t]{2}{*}{ FAMILY/subfamily } & \multirow[t]{2}{*}{ species } & \multicolumn{2}{|l|}{ queen } & \multicolumn{2}{|l|}{ worker } \\
\hline & & Museum & Voucher code & Museum & Voucher code \\
\hline \multicolumn{6}{|l|}{ FORMICIDAE } \\
\hline Aenictinae & Aenictus vaucheri/binghami & MSNG & CASENT0903754 & $\mathrm{AMNH}$ & RAK0094 \\
\hline Agroecomyrmecinae & Tatuidris tatusia & DADC & CASENT0178881 & $\mathrm{BMNH}$ & RAK0001 \\
\hline Amblyoponinae & Adetomyrma sp & & & $\mathrm{AMNH}$ & RAK0003 \\
\hline Amblyoponinae & Amblyopone australis & ANIC & CASENT0172213 & $\mathrm{AMNH}$ & RAK0005 \\
\hline Amblyoponinae & Amblyopone mercovichi & & & MCZ & RAK0006 \\
\hline Amblyoponinae & Apomyrma stygia & MNHN & CASENT0101445 & MCZ & RAK0083 \\
\hline Amblyoponinae & Concoctio concenta & & & MCZ & RAK0011 \\
\hline Amblyoponinae & Myopopone castanea & ANIC & CASENT0172069 & $\mathrm{AMNH}$ & RAK0012 \\
\hline Amblyoponinae & Mystrium sp & CASC & CASENT0104559 & CASC & CASENT0076622 \\
\hline Amblyoponinae & Onychomyrmex doddi & & & $\mathrm{AMNH}$ & RAK0014 \\
\hline Amblyoponinae & Prionopelta punctulata & ANIC & CASENT0172312 & $\mathrm{AMNH}$ & RAK0016 \\
\hline Amblyoponinae & Stigmatomma armigera & & & $\mathrm{AMNH}$ & RAK0004 \\
\hline Amblyoponinae & Stigmatomma pallipes & $A B S$ & CASENT0103553 & MCZ & RAK0009 \\
\hline Amblyoponinae & Stigmatomma pluto & & & $\mathrm{MCZ}$ & RAK0010 \\
\hline Amblyoponinae & Xymmer muticus & & & MCZ & RAK0007 \\
\hline Aneuretinae & Aneuretus simoni & ANIC & CASENT0172259 & MCZ & RAK0074 \\
\hline Cerapachyinae & Acanthostichus serratulus & & & $\mathrm{AMNH}$ & RAK0095 \\
\hline Cerapachyinae & Cerapachys nitidulus & RAKC & RAK127 & $\mathrm{AMNH}$ & RAK0096 \\
\hline Cerapachyinae & Cerapachys doryloides & & & $\mathrm{AMNH}$ & RAK0097 \\
\hline Cerapachyinae & Cylindromyrmex brevitarsus & JTLC & CASENT0610653 & $\mathrm{AMNH}$ & RAK0098 \\
\hline Cerapachyinae & Simopone schoutedeni & & & $\mathrm{AMNH}$ & RAK0099 \\
\hline Dolichoderinae & Dolichoderus bispinosus & ALWC & CASENT0173835 & ALWC & CASENT0173833 \\
\hline Dolichoderinae & Iridomyrmex lividus & ANIC & CASENT0172066 & ANIC & CASENT0172041 \\
\hline Dolichoderinae & Leptomyrmex pallens & & & $\mathrm{AMNH}$ & RAK0075 \\
\hline Dolichoderinae & Tapinoma erraticum & CASC & CASENT0173200 & $\mathrm{AMNH}$ & RAK0078 \\
\hline Dolichoderinae & Technomyrmex albipes & CASC & CASENT0060419 & $\mathrm{AMNH}$ & RAK0079 \\
\hline Dorylinae & Dorylus conradti/helvolus & MSNG & CASENT0903712 & $\mathrm{AMNH}$ & RAK0100 \\
\hline Ecitoninae & Cheliomyrmex morosus & & & $\mathrm{AMNH}$ & RAK0101 \\
\hline Ecitoninae & Eciton hamatum & JTLC & INBIOCRI001283500 & $\mathrm{AMNH}$ & RAK0103 \\
\hline Ecitoninae & Labidus coecus & & & $\mathrm{AMNH}$ & RAK0102 \\
\hline Ectatomminae & Ectatomma tuberculatum & JTLC & JTLC000014186 & $\mathrm{AMNH}$ & RAK0017 \\
\hline Ectatomminae & Gnamptogenys annulata & & & $\mathrm{AMNH}$ & RAK0018 \\
\hline Ectatomminae & Gnamptogenys striatula & MIZA & CASENT0178660 & $\mathrm{AMNH}$ & RAK0019 \\
\hline Ectatomminae & Gnamptogenys bufonis & & & MCZ & RAK0020 \\
\hline Ectatomminae & Gnamptogenys minuta & & & MCZ & RAK0021 \\
\hline Ectatomminae & Rhytidoponera metallica & ANIC & CASENT0172346 & ANIC & CASENT0172345 \\
\hline Ectatomminae & Typhlomyrmex pusillus & MIZA & CASENT0178662 & $\mathrm{AMNH}$ & RAK0023 \\
\hline Ectatomminae & Typhlomyrmex rogenhoferi & & & $\mathrm{AMNH}$ & RAK0024 \\
\hline Formicinae & Formica sp. (fusca group) & CASC & CASENT0173171 & $\mathrm{AMNH}$ & RAK0080 \\
\hline Formicinae & Lasius flavus & CASC & CASENT0173149 & UCDC & CASENT0005406 \\
\hline Formicinae & Oecophylla smaragdina & CASC & CASENT0173644 & $\mathrm{AMNH}$ & RAK0082 \\
\hline
\end{tabular}

Table 2. Continued on next page 
Table 2. Continued

FAMILY/subfamily species queen worker

\begin{tabular}{|c|c|c|c|c|c|}
\hline & & Museum & Voucher code & Museum & Voucher code \\
\hline Formicinae & Polyergus sp & RAKC & RAK0129 & RAKC & RAK0130 \\
\hline Formicinae & Polyrhachis revoili & CASC & CASENT0403971 & CASC & CASENT0227558 \\
\hline Heteroponerinae & Acanthoponera minor & & & AMNH & RAK0025 \\
\hline Heteroponerinae & Heteroponera brouni & MCZ & RAK0128 & AMNH & RAK0026 \\
\hline Heteroponerinae & Heteroponera relicta & & & AMNH & RAK0027 \\
\hline Leptanillinae & Leptanilla swani & $\mathrm{AMNH}$ & RAK129 & AMNH & RAK0084 \\
\hline Leptanilloidinae & $\begin{array}{l}\text { Leptanilloides erinys/ } \\
\text { biconstricta }\end{array}$ & UCDC & CASENT0234616 & AMNH & RAK0104 \\
\hline Martialinae & Martialis heureka & & & MZSP & CASENT0106181 \\
\hline Myrmeciinae & Myrmecia gulosa & CASC & CASENT0103309 & CASC & CASENT0103310 \\
\hline Myrmeciinae & Nothomyrmecia macrops & & & AMNH & RAK0086 \\
\hline Myrmicinae & Aphaenogaster fulva & CASC & CASENT0104857 & CASC & CASENT0103585 \\
\hline Myrmicinae & Carebara vidua & CASC & CASENT0260121 & CASC & CASENT0010803 \\
\hline Myrmicinae & Cataulacus wasmanni & CASC & CASENT0498338 & CASC & CASENT0498558 \\
\hline Myrmicinae & Leptothorax pergandei & MCZ & RAK0125 & MCZ & RAK0126 \\
\hline Myrmicinae & Manica rubida & & & AMNH & RAK0090 \\
\hline Myrmicinae & Messor barbarus & RAKC & RAK0123 & RAKC & RAK0124 \\
\hline Myrmicinae & Metapone madagascarica & CASC & CASENT0004524 & MCZ & RAK0093 \\
\hline Myrmicinae & Monomorium pharaonis & ABS & CASENT0104094 & ABS & CASENT0104095 \\
\hline Myrmicinae & Myrmica wheeleri & $\mathrm{MCZ}$ & CASENT0102860 & $\mathrm{MCZ}$ & CASENT0102862 \\
\hline Myrmicinae & $\begin{array}{l}\text { Pogonomyrmex } \\
\text { uruguayensis }\end{array}$ & RAJC & CASENT0172689 & RAJC & CASENT0103054 \\
\hline Paraponerinae & Paraponera clavata & RAKC & RAK0122 & AMNH & RAK0028 \\
\hline Ponerinae & Anochetus mayri & $A B S$ & CASENT0103555 & MCZ & CASENT0003324 \\
\hline Ponerinae & Asphinctopone silvestrii & & & MCZ & RAK0031 \\
\hline Ponerinae & Belonopelta deletrix & & & $\mathrm{MCZ}$ & RAK0032 \\
\hline Ponerinae & Bothroponera pachyderma & & & AMNH & RAK0054 \\
\hline Ponerinae & Brachyponera croceicornis & & & AMNH & RAK0051 \\
\hline Ponerinae & Centromyrmex brachycola & UCDC & CASENT0178343 & $\mathrm{AMNH}$ & RAK0033 \\
\hline Ponerinae & Cryptopone gilva & CASC & CASENT0006055 & AMNH & RAK0034 \\
\hline Ponerinae & Diacamma ceylonense & & & AMNH & RAK0035 \\
\hline Ponerinae & Dinoponera lucida & & & AMNH & RAK0036 \\
\hline Ponerinae & Dolioponera fustigera & & & $\mathrm{MCZ}$ & RAK0037 \\
\hline Ponerinae & Emeryopone buttelreepeni & & & $\mathrm{MCZ}$ & RAK0038 \\
\hline Ponerinae & Hagensia marleyi & & & $\mathrm{MCZ}$ & RAK0053 \\
\hline Ponerinae & Harpegnathos saltator & & & $\mathrm{AMNH}$ & RAK0039 \\
\hline Ponerinae & Hypoponera sp1. & & & $\mathrm{AMNH}$ & RAK0040 \\
\hline Ponerinae & $\begin{array}{l}\text { Leptogenys (Leptogenys) } \\
\text { sp.1 }\end{array}$ & & & $\mathrm{AMNH}$ & RAK0041 \\
\hline Ponerinae & Leptogenys (Lobopelta) sp.2 & & & $\mathrm{AMNH}$ & RAK0042 \\
\hline Ponerinae & Leptogenys podenzanai & & & $\mathrm{MCZ}$ & RAK0043 \\
\hline Ponerinae & Loboponera obeliscata & & & AMNH & RAK0044 \\
\hline Ponerinae & Loboponera vigilans & & & $\mathrm{AMNH}$ & RAK0045 \\
\hline Ponerinae & Myopias chapmani & ANIC & CASENT0172094 & ANIC & CASENT01 \\
\hline
\end{tabular}

Table 2. Continued on next page 
Table 2. Continued

\begin{tabular}{|c|c|c|c|c|c|}
\hline FAMILY/subfamily & species & queen & & worker & \\
\hline & & Museum & Voucher code & Museum & Voucher code \\
\hline Ponerinae & Neoponera apicalis & ALWC & CASENT0103060 & $\mathrm{AMNH}$ & RAK0048 \\
\hline Ponerinae & Neoponera villosa & & & $\mathrm{AMNH}$ & RAK0058 \\
\hline Ponerinae & Odontomachus bauri & CASC & CASENT0172630 & $\mathrm{AMNH}$ & RAK0030 \\
\hline Ponerinae & Odontoponera transversa & $\mathrm{BMNH}$ & CASENT0900664 & $\mathrm{AMNH}$ & RAK0047 \\
\hline Ponerinae & Ophthalmopone berthoudi & & & MCZ & RAK0049 \\
\hline Ponerinae & Pachycondyla crassinoda & & & $\mathrm{AMNH}$ & RAK0050 \\
\hline Ponerinae & Cryptopone guianensis & & & MCZ & RAK0052 \\
\hline Ponerinae & Pseudoneoponera porcata & & & $\mathrm{AMNH}$ & RAK0055 \\
\hline Ponerinae & Pseudoponera stigma & & & $\mathrm{AMNH}$ & RAK0056 \\
\hline Ponerinae & Paltothyreus tarsatus & & & $\mathrm{AMNH}$ & RAK0057 \\
\hline Ponerinae & Phrynoponera gabonensis & & & $\mathrm{AMNH}$ & RAK0059 \\
\hline Ponerinae & Platythyrea punctata & ABS & CASENT0104429 & $\mathrm{AMNH}$ & RAK0060 \\
\hline Ponerinae & Platythyrea turneri & & & MCZ & RAK0061 \\
\hline Ponerinae & Plectroctena strigosa & & & $\mathrm{AMNH}$ & RAK0062 \\
\hline Ponerinae & Ponera alpha & & & MCZ & RAK0063 \\
\hline Ponerinae & Ponera pennsylvanica & CASC & CASENT0006086 & $\mathrm{AMNH}$ & RAK0064 \\
\hline Ponerinae & Psalidomyrmex procerus & & & $\mathrm{AMNH}$ & RAK0065 \\
\hline Ponerinae & Simopelta oculata & & & MCZ & RAK0066 \\
\hline Ponerinae & Streblognathus peetersi & & & $\mathrm{AMNH}$ & RAK0067 \\
\hline Ponerinae & Thaumatomyrmex atrox & & & $\mathrm{AMNH}$ & RAK0068 \\
\hline Proceratiinae & Discothyrea oculata & & & $\mathrm{AMNH}$ & RAK0069 \\
\hline Proceratiinae & Discothyrea testacea & ABS & CASENT0103848 & $\mathrm{AMNH}$ & RAK0070 \\
\hline Proceratiinae & Proceratium croceum & ABS & CASENT0104440 & $\mathrm{AMNH}$ & RAK0071 \\
\hline Proceratiinae & Proceratium pergandei & & & $\mathrm{AMNH}$ & RAK0072 \\
\hline Proceratiinae & Probolomyrmex guineensis & & & $\mathrm{AMNH}$ & RAK0073 \\
\hline Pseudomyrmecinae & Pseudomyrmex gracilis & ABS & CASENT0103779 & $\mathrm{AMNH}$ & RAK0087 \\
\hline Pseudomyrmecinae & Tetraponera aethiops & & & $\mathrm{AMNH}$ & RAK0088 \\
\hline Pseudomyrmecinae & Tetraponera attenuata & CASC & CASENT0217587 & $\mathrm{AMNH}$ & RAK0089 \\
\hline Sphecomyrminae $^{\dagger}$ & Sphecomyrma freyi ${ }^{\dagger}$ & & & $\mathrm{AMNH}$ & AMNH NJ-943 \\
\hline SCOLIIDAE & Scolia nobilitata & $\mathrm{AMNH}$ & RAK0121 & & \\
\hline VESPIDAE & Metapolybia cingulata & $\mathrm{AMNH}$ & RAK0120 & & \\
\hline
\end{tabular}

Information on museum holdings and voucher codes for queens and workers. ABS, Archbold Biological Station; ALWC, Alexander Wild Collection; AMNH, American Museum of Natural History; ANIC, Australian National Insect Collection; BMNH, British Museum of Natural History; CASC, California Academy of Science; DADC, David A. Donoso Collection; JTLC, Jack Longino Collection; MCZ, Museum of Comparative Zoology (Harvard); MIZA, Museo del Instituto de. Zoología Agrícola (Venezuela); MNHN, Muséum national d'Histoire naturelle; MSNG, Natural History Museum, Genoa; MZSP, Museu de Zoologia Universidade de São Paulo; RAJC, Robert Johnson Collection; RAKC, Roberto Keller Collection; UCDC; University of California Davis.

${ }^{\dagger}$ denotes extinct taxa.

DOI: 10.7554/eLife.01539.010

\section{Queen morphology reflects colony foundation strategy}

Out of the two morphological categories of queens we identified, one is closer to workers in size of T1 vs T2 (Figure 1B). Similarly for behavior, it is known that queens in some species go through a workerlike phase after they mate and shed their wings. In several lineages, lone founding queens regularly forage outside the nest (they are 'non-claustral'), and can hunt and carry large prey to feed the first 
Table 3. Queen thoracic morphology and type of colony foundation across ants

\begin{tabular}{|c|c|c|c|c|c|}
\hline Subfamily & Genus & $\begin{array}{l}\text { T1/T2 in } \\
\text { queens }\end{array}$ & $\mathrm{T} 1$ in queens & $\begin{array}{l}\text { Colony } \\
\text { founding }\end{array}$ & References \\
\hline Aenictinae & Aenictus & 2.742 & intermediate* & fission & $\begin{array}{l}\text { (Gotwald and } \\
\text { Cunningham-van } \\
\text { Someren, 1976) }\end{array}$ \\
\hline Agroeconomyrmecinae & Tatuidris & 0.111 & reduced & unknown & \\
\hline Amblyoponinae & Amblyopone & 0.382 & intermediate & non-claustral & $\begin{array}{l}\text { (Haskins and } \\
\text { Haskins, 1951) }\end{array}$ \\
\hline Amblyoponinae & Apomyrma & 0.338 & intermediate & unknown & \\
\hline Amblyoponinae & Myopopone & 0.453 & intermediate & non-claustral & (Ito, 2010) \\
\hline Amblyoponinae & Mystrium & 0.454 & intermediate & non-claustral & $\begin{array}{l}\text { (Molet et al., } \\
\text { 2009) }\end{array}$ \\
\hline Amblyoponinae & Prionopelta & 0.514 & intermediate & non-claustral & $\begin{array}{l}\text { (Ito and Billen, } \\
\text { 1998) }\end{array}$ \\
\hline Aneuretinae & Aneuretus & 0.096 & reduced & claustral & $\begin{array}{l}\text { (Wilson et al., } \\
\text { 1956) }\end{array}$ \\
\hline Cerapachyinae & Cerapachys & 0.364 & intermediate & $\begin{array}{l}\text { unknown ICF + } \\
\text { fission }\end{array}$ & (Brown, 1975) \\
\hline Cerapachyinae & Cylindromyrmex & 0.454 & intermediate & non-claustral & $\begin{array}{l}\text { (Delabie and Reis, } \\
2000 \text { ) }\end{array}$ \\
\hline Dolichoderinae & Dolichoderus & 0.061 & reduced & unknown & \\
\hline Dolichoderinae & Iridomyrmex & 0.071 & reduced & claustral & $\begin{array}{l}\text { (Hölldobler and } \\
\text { Carlin, 1985) }\end{array}$ \\
\hline Dolichoderinae & Tapinoma & 0.111 & reduced & claustral & $\begin{array}{l}\text { (Kannowski, } \\
\text { 1959) }\end{array}$ \\
\hline Dolichoderinae & Technomyrmex & 0.071 & reduced & claustral & $\begin{array}{l}\text { (Yamauchi et al., } \\
\text { 1991) }\end{array}$ \\
\hline Dorylinae & Dorylus & 0.372 & intermediate* & fission & $\begin{array}{l}\text { (Kronauer et al., } \\
2004 \text { ) }\end{array}$ \\
\hline Ecitoninae & Eciton & 0.469 & intermediate* & fission & (Schneirla, 1949) \\
\hline Ectatomminae & Ectatomma & 0.325 & intermediate & non-claustral & $\begin{array}{l}\text { (Dejean and } \\
\text { Lachaud, 1992) }\end{array}$ \\
\hline Ectatomminae & Gnamptogenys & 0.331 & intermediate & non-claustral & ( $\ddagger$ \\
\hline Ectatomminae & Rhytidoponera & 0.363 & intermediate & non-claustral & (Ward, 1981) \\
\hline Ectatomminae & Typhlomyrmex & 0.504 & intermediate & unknown & \\
\hline Formicinae & Formica & 0.076 & reduced & claustral & (Stille, 1996) \\
\hline Formicinae & Lasius & 0.053 & reduced & claustral & (Stille, 1996) \\
\hline Formicinae & Oecophylla & 0.066 & reduced & claustral & $\begin{array}{l}\text { (Hölldobler and } \\
\text { Wilson, 1978) }\end{array}$ \\
\hline Formicinae & Polyergus & 0.323 & intermediate & non-claustral† & (Mori et al., 1995) \\
\hline Formicinae & Polyrhachis & 0.072 & reduced & $\begin{array}{l}\text { claustral and } \\
\text { non-claustral }\end{array}$ & $\begin{array}{l}\text { (Lenoir and } \\
\text { Dejean, 1994) }\end{array}$ \\
\hline Heteroponerinae & Heteroponera & 0.485 & intermediate & non-claustral & (§) \\
\hline Leptanillinae & Leptanilla & 2.685 & intermediate* & fission & (Masuko, 1990) \\
\hline Leptanilloidinae & Leptanilloides & 3.021 & intermediate* & fission & $\begin{array}{l}\text { (Donoso et al., } \\
\text { 2006) }\end{array}$ \\
\hline Martialinae & Martialis & $\mathrm{n} / \mathrm{a}$ & unknown & unknown & \\
\hline Myrmeciinae & Myrmecia & 0.485 & intermediate & non-claustral & $\begin{array}{l}\text { (Haskins and } \\
\text { Haskins, 1950) }\end{array}$ \\
\hline Myrmicinae & Aphaenogaster & 0.117 & reduced & claustral & (Lubertazzi, 2012) \\
\hline
\end{tabular}

Table 3. Continued on next page 
Table 3. Continued

\begin{tabular}{|c|c|c|c|c|c|}
\hline Subfamily & Genus & $\begin{array}{l}\mathrm{T} 1 / \mathrm{T} 2 \text { in } \\
\text { queens }\end{array}$ & T1 in queens & $\begin{array}{l}\text { Colony } \\
\text { founding }\end{array}$ & References \\
\hline Myrmicinae & Carebara & 0.072 & reduced & claustral & $\begin{array}{l}\text { (Robertson and } \\
\text { Villet, 1989) }\end{array}$ \\
\hline Myrmicinae & Cataulacus & 0.494 & intermediate & unknown & \\
\hline Myrmicinae & Leptothorax & 0.090 & reduced & claustral & $\begin{array}{l}\text { (Keller and } \\
\text { Passera, 1989) }\end{array}$ \\
\hline Myrmicinae & Messor & 0.110 & reduced & $\begin{array}{l}\text { claustral and } \\
\text { non-claustral }\end{array}$ & (Brown, 1999) \\
\hline Myrmicinae & Metapone & 0.428 & intermediate & unknown & \\
\hline Myrmicinae & Monomorium & 0.132 & reduced & claustral & (Bolton, 1986) \\
\hline Myrmicinae & Myrmica & 0.071 & reduced & $\begin{array}{l}\text { claustral and } \\
\text { non-claustral }\end{array}$ & $\begin{array}{l}\text { (Brown and } \\
\text { Bonhoeffer, 2003) }\end{array}$ \\
\hline Myrmicinae & Pogonomyrmex & 0.097 & reduced & $\begin{array}{l}\text { claustral and } \\
\text { non-claustral }\end{array}$ & (Johnson, 2002) \\
\hline Paraponerinae & Paraponera & 0.086 & reduced & non-claustral & (\#) \\
\hline Ponerinae & Anochetus & 0.367 & intermediate & non-claustral & (Brown, 1978) \\
\hline Ponerinae & Centromyrmex & 0.493 & intermediate & non-claustral & $\begin{array}{l}\text { (Dejean and } \\
\text { Fénéron, 1996) }\end{array}$ \\
\hline Ponerinae & Cryptopone & 0.533 & intermediate & non-claustral & (Peeters, 1997) \\
\hline Ponerinae & Ponera & 0.356 & intermediate & non-claustral & $\begin{array}{l}\text { (Kannowski, } \\
\text { 1959) }\end{array}$ \\
\hline Ponerinae & Myopias & 0.282 & intermediate & non-claustral & (Peeters, 1997) \\
\hline Ponerinae & Odontomachus & 0.411 & intermediate & non-claustral & (Brown, 1976) \\
\hline Ponerinae & Odontoponera & 0.524 & intermediate & non-claustral & (Peeters, 1997) \\
\hline Ponerinae & Pachycondyla & 0.385 & intermediate & non-claustral & (Peeters, 1997) \\
\hline Ponerinae & Platythyrea & 0.417 & intermediate & non-claustral & (Peeters, 1997) \\
\hline Proceratiinae & Discothyrea & 0.093 & reduced & $\begin{array}{l}\text { non-claustral } \\
\text { and claustral }\end{array}$ & $\begin{array}{l}\text { (Dejean and } \\
\text { Dejean, 1998) }\end{array}$ \\
\hline Proceratiinae & Proceratium & 0.095 & reduced & non-claustral & (१) \\
\hline Pseudomyrmecinae & Pseudomyrmex & 0.479 & intermediate & non-claustral & $(* \star)$ \\
\hline Pseudomyrmecinae & Tetraponera & 0.558 & intermediate & non-claustral & $(* \star)$ \\
\hline Sphecomyrminae ${ }^{\dagger}$ & Sphecomyrma ${ }^{\dagger}$ & $\mathrm{n} / \mathrm{a}$ & unknown & unknown & \\
\hline \multicolumn{6}{|l|}{ OUTGROUPS } \\
\hline Scoliinae & Scolia & 0.087 & reduced & non-social & $(† \dagger)$ \\
\hline Polistinae & Metapolybia & 0.074 & reduced & fission & $(† \dagger)$ \\
\hline
\end{tabular}

Queen thoracic morphology and type of colony foundation across ants. The wasp taxa Scolia and Metapolybia are included as outgroups.

*species with wingless queens. ${ }^{\dagger}$ denotes extinct taxa.

†Polyergus is an obligatory social parasite of Formica spp.

łJohn Lattke, personal communication.

§Rodrigo Feitosa, personal communication.

\#Haskins CP, Enzmann EV (1937) Studies of certain sociological and physiological features in the Formicidae. Ann NY Acad Scien 37:97-162; Michael Breed, personal communication.

१Fuminori Ito, personal communication; Keiichi Masuko, personal communication.

**Philip Ward, personal communication.

††James M Carpenter, personal communication.

DOI: 10.7554/eLife.01539.011 


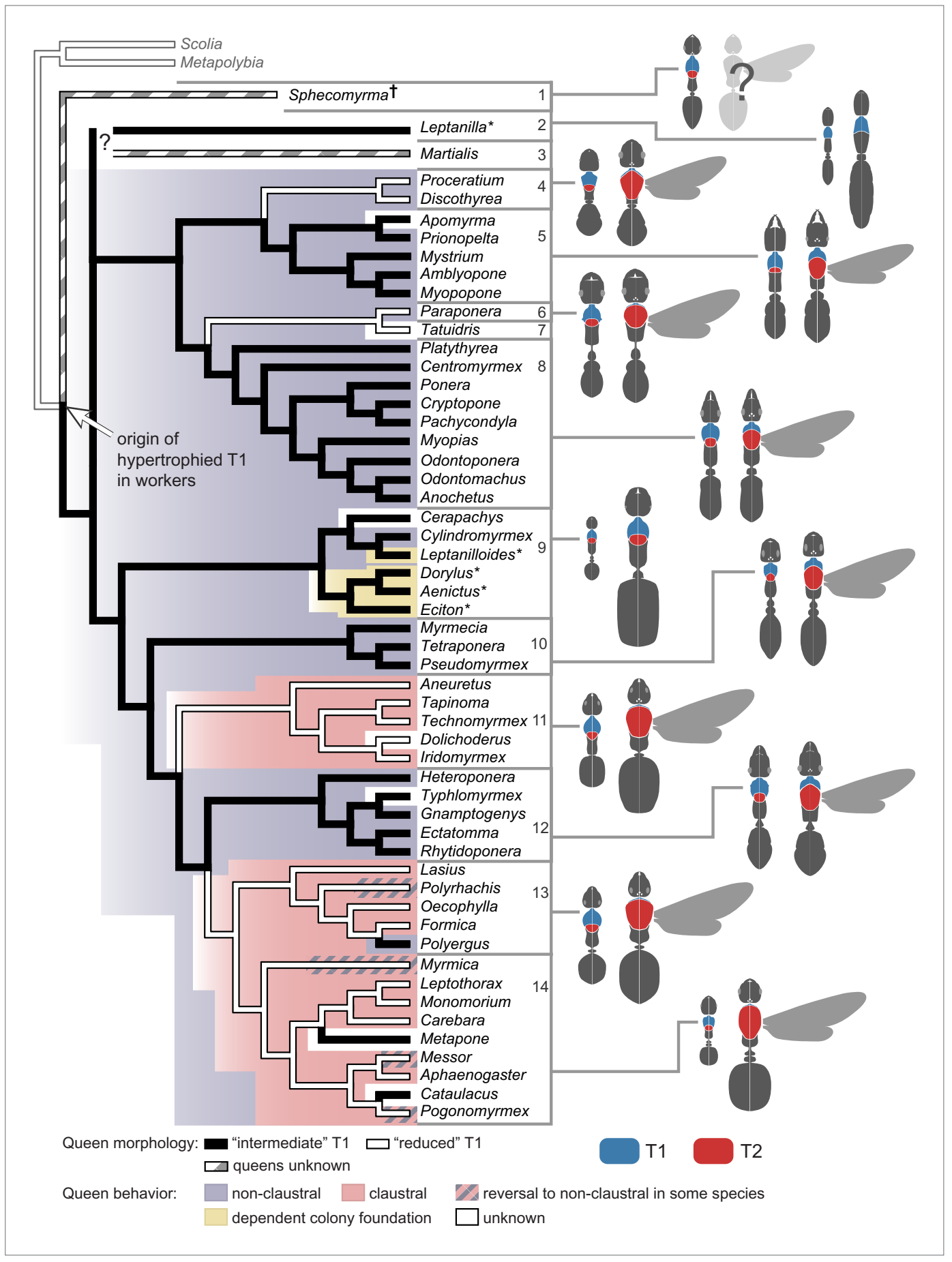

Figure 3. Phylogenetic reconstruction reveals a single origin of a hypertrophied $T 1$ in workers and multiple independent origins of 'reduced' T1 in queens. The latter is associated with modifications in modes of colony foundation. Tree branches and tree background are colored for queen morphology and founding behavior respectively, according to the parsimony ancestral reconstruction. Typical queen-worker dimorphism shown to the right to illustrate ratio T1/T2 (not to scale). Species with wingless queens are marked with an asterisk. Phylogeny was pruned from Moreau et al. (2006). Placement of Sphecomyrma ${ }^{\dagger}$ and Martialis after Grimaldi et al. (1997) and Rabeling et al. (2008), respectively. Metapolybia and Scolia wasps are included as outgroups. Data on the species are analyzed, and their morphology and type of colony founding behavior are summarized in Table 3. Numbers correspond to major taxonomic groups within Formicidae after Ward (2007): 1, Sphecomyrminae ; 2, Leptanillinae; 3, Martialinae; 4, Proceratiinae; 5, Amblyoponinae; 6, Paraponerinae; 7, Agroecomyrmecinae; 8, Ponerinae; 9, dorylomorphs; 10, myrmeciomorphs; 11, dolichoderomorphs; 12, ectaheteromorphs; 13, Formicinae; 14, Myrmicinae.

DOI: 10.7554/eLife.01539.012 
generation of worker larvae (Haskins, 1970; Peeters, 1997). During several weeks, these non-claustral queens behave much like workers. This contrasts with the vast majority of ants, where founding queens are confined to the nest (they are 'claustral') and, instead of foraging, use their metabolic reserves to feed their first brood (Hölldobler and Wilson, 1990; Wheeler and Buck, 1996).

To test the hypothesis that the morphological classes associate with the behavioral classes, we compiled data on mode of colony foundation for the 54 ant species in our tree (Figure 3; Table 3). We could find information for a total of 45 species: 25 non-claustral, 15 claustral, and 4 with dependent colony foundation (i.e. colony fission, when queens are never alone). Unfortunately, there are no data for some of the putative early lineages (the fossil Sphecomyrma [Grimaldi et al., 1997], and the two rare subfamilies Leptanillinae and Martialinae [Borowiec et al., 2011]). Using parsimony and ML methods, we established that non-claustral behavior is the most likely ancestral condition (Figure 3; ML proportional likelihood $=0.919$ ). Claustral colony foundation has evolved at least twice independently, with reversals to non-claustral foundation occurring sporadically within some genera. Our reconstruction supports colony fission as a secondary shift among ants (Cronin et al., 2013).

Next, we performed a Concentrated Changes test (Maddison, 1990) to investigate the phylogenetic correlation between queen thoracic morphology and founding behavior. We found strong support for correlated evolution ( $p=0.027$, calculated by simulation of 100,000 actual changes with two gains and four loses): all queens with an 'intermediate T1' are non-claustral founders, whereas two of four independent origins of queens with a 'reduced T1' coincide with shifts to claustral foundation (Figure 3; clades 11 and 13+14). A reversal in morphology to 'intermediate $T 1$ ' corresponds to a modified claustral behavior in Polyergus (clade 13) which parasitizes colonies of Formica, hence Polyergus queens need to fight to invade the host colonies (Trager, 2013). However, sporadic reversals to non-claustral founding have been reported for a few species (Lenoir and Dejean, 1994; Brown, 1999; Johnson, 2002; Brown and Bonhoeffer, 2003) that according to our morphological survey are not accompanied by reversals in queen morphology (Figure 3). Modeling suggests that such facultative reversals to non-claustral behavior are likely to occur in cases of increased resource availability (Johnson, 2002; Brown and Bonhoeffer, 2003). We did not observe changes in the T1/T2 ratio in lineages that secondarily evolved colony fission, even though this mode of colony foundation is known to co-occur with wing-loss in queens (Cronin et al., 2013). This suggests that, despite being wingless, in the absence of the selective pressures related to worker-like foraging (as in non-claustral queens) or of the need for storing metabolic reserves as flight muscles (as in claustral queens), queens in those lineages maintain the ancestral T1/T2 ratio (e.g. dorylomorph clade in Figure 3).

\section{Discussion}

The ecological dominance of ants in terrestrial ecosystems is unparalleled in the animal kingdom (Wilson, 1971; Folgarait, 1998). Because no other group of social insects reaches equivalent levels of adaptive radiation and species-richness (Hölldobler and Wilson, 1990), it seems that factors in addition to social behavior and division of labor promoted ant diversification. The evolutionary success of ants is indisputably associated with a strong divergence between queens and workers. A caste of flightless workers specialized in non-reproductive activities is unique among social Hymenoptera. However, rather than being just simplified, wingless versions of the queen, the thorax of ant workers has its own specialization. Relative to the thoracic morphology of queens, which is typical of species of flying insects, worker ants have an unusually large T1 and T1-associated muscles, which provide superior strength and mobility to the neck controlling head movements.

Control of the head is of great importance for ant workers, which in some species singly hunt and carry prey up to 30-90 times their weight (Dejean, 2011; Dejean et al., 2012). Among insects, ant carrying behavior is unique in that workers lift their load off the ground. Many other insects can move relatively large objects, but by dragging (e.g., spider wasps) or rolling them (e.g., dung beetles) on the ground, or holding them while flying (e.g., robber flies). Biomechanical studies on grass-cutting ants have shown that workers perform controlled head movements at the neck articulation when transporting large objects (Moll et al., 2010). Precise head movements are essential to reduce displacement of the center of mass, and retain stability while carrying objects many times the workers' weight and length. Our finding that worker ants differ from queens and other flying insects in the configuration and size of the T1-associated muscles suggests that ants can achieve this biomechanical feat by virtue of their specialized neck musculature. This represents a striking structural innovation, differentiating ant workers from the typical flying insects, which had not been recognized until now. Their distinctive internal 
skeletomuscular modifications presumably enhance their behavior as flightless foragers and heavyload transporters. We propose that the modified T1 was an innovation that helped ants to use their heads and mandibles in novel ways, and hence exploit a broader spectrum of trophic resources. Compared to social bees and wasps (Hölldobler and Wilson, 1990), where worker morphology is constrained by the requirements of a winged thorax, mandibular morphology and function have specialized enormously across ant lineages (Paul and Gronenberg, 1999), in parallel with their much greater diversification of foraging habits.

Our analysis also showed that queens fall in two distinct anatomical types that evolved in association with the two strategies of independent colony founding. Foraging activity during independent foundation is high in non-claustral species vs absent in claustral species. Non-claustral queens have a T1 that is closer in size to that of workers, while claustral queens, which do not go through a workerlike phase, have a much more reduced T1. Unfortunately, biomechanical data of neck strength in queens are difficult to obtain because they are evasive and, especially in claustral species, cannot be induced to carry objects. Claustral queens have an enlarged T2 relative to non-claustral queens, reflecting the existence of massive wing muscles (Figure 2A). A correlation between increased wing muscle mass and claustral behavior has been suggested before: these larger muscles do not function to enhance flight, rather they are a solution for storing amino acids that are essential for feeding the first generation of workers without outside foraging (Jones et al., 1978; Peeters, 2012) . We speculate that, during the acquisition of claustral behavior, the decrease in foraging activity lessened the constraint on the size of T1, thus allowing T2 to expand and accommodate larger wing muscles as metabolic reserves. Differences in the nesting habits of queens, such as excavating a nest vs nesting in pre-existing cavities, might also impose variable muscle requirements. However, this type of behavioral differences occurs across species in a scattered pattern that does not match the anatomical categories we revealed. There are examples of nest excavating by queens with 'intermediate' (e.g., Amblyopone) and 'reduced' T1 (e.g., Pogonomyrmex), and of nesting in pre-existing cavities by 'intermediate' (e.g., Tetraponera) as well as 'reduced' T1 (e.g., Leptothorax) species.

While data on queen morphology is readily accessible from museum collections for many species, knowledge about their founding behavior remains sparse. There is no published information in many important genera, possibly because this requires field observations of behavior at an appropriate time of the year. Our findings provide a means of predicting colony foundation strategy from the morphology of the queen thorax, and thus guide field research on particular species of interest. For example, within the subfamily Myrmicinae (clade 14 in Figure 3), the genera Cataulacus and Metapone show independent reversals to an 'intermediate $\mathrm{T} 1$ ' in queens, suggesting that colony foundation is not claustral as in closely related genera. Importantly, the phylogenetic component of our correlation provides a powerful tool to infer the ecology of extinct clades for which behavioral observations are impossible. For example, we lack data on queens of two early lineages, the extinct Sphecomyrma ${ }^{\dagger}$ and the enigmatic Martialis, but based on our reconstructions we can predict that they will have an 'intermediate $\mathrm{T} 1$ ' and behave non-claustrally.

Our finding that the ratio of the lengths of T1 and T2 is inverted between queens and workers suggests that a morphological trade-off was at play in determining the relative size of these two segments. It is likely that T1 can become hypertrophied only at the expense of a reduced, non-functional T2. Indeed, our anatomical analysis showed that some of the internal modifications of T1 in workers are only possible in conjunction with a complete absence of wing muscles. Conversely, only queens with a highly reduced T1 have an expanded T2 that constitutes most of the thoracic dorsum (queens with intermediate T1 are also intermediate for T2, see Figure 1B). This morphological trade-off between adjacent body segments can occur due to competition for metabolic resources during preadult development (Nijhout and Emlen, 1998). It is possible that the functional cost of enlarging T2 (reserves for colony founding) at the expense of T1 (reduced neck strength and work performance), occurred when founding behavior gradually shifted to claustral, with a decreased need to forage outside the nest.

\section{Materials and methods}

\section{Phylogenetic sampling}

We compared the thorax of queens and workers across multiple species representing all major ant lineages. First, we measured the length of the thoracic segments and entire thorax in a sample of 
individuals belonging to 11 different species from five ant subfamilies (Table 1). Second, we used a database of scanning electronic micrographs $(K e l l e r, 2011)$ and an online database of light microscopy images (http://www.antweb.org) to further assess the extent of the taxonomic distribution of the traits of interest. We inspected the external thorax of workers belonging to 110 ant species, and of queens belonging to a subset of 47 species where this caste is known or available (listed in Table 2). This taxon sampling represents all 21 extant subfamilies with the exception of the dorylomorph subfamily Aenictogitoninae (for which only males have been formally described), and includes the extinct subfamily Sphecomyrminae ${ }^{\dagger}$. Lastly, we analyzed internal thoracic anatomy by dissecting multiple individuals from 19 representative species (Table 1). Our sample of queens included individuals both before and after the phase of muscle reabsorption, as assessed by the shedding of their wings.

\section{Morphometrics and anatomical analysis}

For the quantitative characterization of the thorax, we took dorsal and lateral photographs of pinned specimens (Museum of Comparative Zoology, Harvard University) with a JVC digital camera mounted on a Leica MZ16 binocular microscope (images are deposited in the Dryad data repository under DOI doi: 10.5061/dryad.d62p2/2). We then measured (ImageJ, http://rsb.info.nih.gov/ij) the dorsal length of the first (T1), second (T2), and third (T3) thoracic segments along the midline, and the total thoracic length $(T L)$ as the diagonal length in profile from the anterior-most point of $\mathrm{T} 1$ to the posterior-most point of T3 (Figure 1-figure supplement 1; measurements are available in the Dryad data repository under http://dx.doi.org/10.5061/dryad.d62p2/1).

For the analysis of internal anatomy we performed muscle preparations using specimens fixed in either $80 \%$ ethanol or $4 \%$ formaldehyde, and sectioning their thoraces in sagittal and parasagittal planes or disarticulating the plates of the thorax. Muscle preparations were stained in $0.2 \%$ methylene blue (Sigma-Aldrich) to increase contrast against other tissues. We also performed skeletal preparations by disarticulating specimens with overnight digestion of soft tissues in $10 \% \mathrm{KOH}$, and kept in $90 \%$ ethanol for inspection. When necessary (i.e., lightly pigmented specimens), skeletal preparations were stained in $70 \%$ ethanol saturated solution of Chlorazol Black E (Sigma-Aldrich).

\section{Statistical analysis}

All analyses were performed with $\mathrm{R}$ ( $R$ Development Core Team, 2008). Residuals of the models have been checked for normality and equality of variance, and data have been transformed when necessary. To compare the relative investment in the thoracic plates 1 and 2 between castes, we performed a linear model (LM) constructed as $\sqrt{ }(T 1 / T 2) \sim$ species * caste. T1/T2 is the ratio of the thoracic plate 1 length 'T1' over the length of the thoracic plates 2 ' $\mathrm{T} 2$ '. ' ${ }^{* \prime}$ indicated that the effects were tested for both main factors as well as interaction.

\section{Phylogenetic mapping and correlation of queen behavior and morphology}

We scored the queens of our 56 exemplar species as either 'reduced' or 'intermediate'. We divided the length of T1 by the length of T2, and determined a cut-off index equal to 0.25 based on our previous morphometric analysis. We then assigned 'reduced' to queens falling below the cut-off value and 'intermediate' for queens falling above it. Missing data (i.e., the unknown queens of Sphecomyrma freyi ${ }^{\dagger}$, and Martialis eureka) (Grimaldi et al., 1997; Rabeling et al., 2008) were coded as '?'. For the modes of colony foundation we assigned states for 'non-claustral', 'claustral' and 'fission' based on records from the scientific literature (Table 3). Unknown mode of colony foundation was coded as '?'. Data in Table 3 correspond to a single queen for each of the species in Figure 1 (except Brachyponera lutea because its exact phylogenetic position within the subfamily Ponerinae remains undetermined) and from 44 more species (listed in Table 2).

Character evolution was reconstructed under parsimony using WinClada (Nixon, 2002) and under maximum likelihood (ML) using Mesquite (Maddison and Maddison, 2012), under the Mk1 model, (Lewis, 2001). Ambiguous optimizations under parsimony were resolved using DELTRAN. This algorithm gave results closer to the ML analysis than did the ACCTRAN parsimony algorithm. Tree topology with branch lengths was pruned from Moreau et al. (2006). We implemented the concentrated changes test (Maddison, 1990), using MacClade (Maddison and Maddison, 2005), to test for a correlation between modes of colony foundation and queen morphology. This test calculates the probability that changes in a binary character along the phylogeny are distributed randomly on the branches defined by a second binary character. We therefore transformed our data on behavior and morphology to 
binary characters by pruning out the branches with fission and wingless queens, since both traits always co-occur in the phylogeny.

\section{Acknowledgements}

We thank Stefan Cover and Gary Alpert for access to the MCZ collection and imaging respectively (Cambridge, USA), and David Duneau for advice on statistical analysis. The comments made by the editor and three reviewers helped to improve our manuscript.

\section{Additional information}

Funding

\begin{tabular}{lll} 
Funder & Grant reference number & Author \\
\hline $\begin{array}{l}\text { Portuguese Science and Technology } \\
\text { Foundation (FCT) }\end{array}$ & SFRH/BPD/65529/2009 & Roberto A Keller \\
\hline $\begin{array}{l}\text { Portuguese Science and Technology } \\
\text { Foundation (FCT) }\end{array}$ & PTDC/BIA-BEC/100243/2008 & Patrícia Beldade \\
\hline $\begin{array}{ll}\text { Oeiras Municipality Installation Grant } & \text { Patrícia Beldade }\end{array}$
\end{tabular}

The funders had no role in study design, data collection and interpretation, or the decision to submit the work for publication.

Author contributions

RAK, Conception and design, Acquisition of data, Analysis and interpretation of data, Drafting or revising the article; $\mathrm{CP}$, Conception and design, Analysis and interpretation of data, Drafting or revising the article, Contributed unpublished essential data or reagents; PB, Analysis and interpretation of data, Drafting or revising the article

\section{Additional files}

Major dataset

The following dataset was generated:

\begin{tabular}{lllll}
\hline Author(s) & Year & Dataset title & Dataset ID and/or URL & $\begin{array}{l}\text { Database, license, and } \\
\text { accessibility information }\end{array}$ \\
\hline $\begin{array}{l}\text { Keller RA, Peeters C, } 2014 \\
\text { Beldade P }\end{array}$ & $\begin{array}{l}\text { Data from: Evolution of thorax } \\
\text { architecture in ant castes } \\
\text { highlights trade-off between }\end{array}$ & $\begin{array}{l}\text { http://dx.doi.org/ } \\
10.5061 / \text { dryad.d62p2 }\end{array}$ & $\begin{array}{l}\text { Data available from the } \\
\text { Dryad Digital Repository. }\end{array}$
\end{tabular}

flight and ground behaviors

\section{References}

Baldwin JM. 1896. A new factor in evolution. The American Naturalist 30:441-451. doi: 10.1086/ 276408.

Beldade P, Mateus ARA, Keller RA. 2011. Evolution and molecular mechanisms of adaptive developmental plasticity. Molecular Ecology 20:1347-1363. doi: 10.1111/j.1365-294X.2011.05016.x.

Bolton B. 1986. Apterous females and shift of dispersal strategy in the Monomorium salomonis-group (Hymenoptera: Formicidae). Journal of Natural History 20:267-272. doi: 10.1080/00222938600770211.

Borowiec ML, Schulz A, Alpert GD, Baňař P. 2011. Discovery of the worker caste and descriptions of two new species of Anomalomyrma (Hymenoptera: Formicidae: Leptanillinae) with unique abdominal morphology. Zootaxa 28:1-14.

Brady SG, Schultz TR, Fisher BL, Ward PS. 2006. Evaluating alternative hypotheses for the early evolution and diversification of ants. Proceedings of the National Academy of Sciences of the United States of America 103:18172-18177. doi: 10.1073/pnas.0605858103.

Brown WL Jnr. 1975. Contributions toward a Reclassification of the Formicidae. V. Ponerinae, Tribes Platythyreini, Cerapachyini, Cylindromyrmecini, Acanthostichini, and Aenictogitini. Search Agric 5:1-116.

Brown WL Jnr. 1976. Contributions toward a reclassification of the Formicidae. Part VI. Ponerinae, tribe Ponerini, subtribe Odontomachiti. Section A. Introduction, subtribal characters. Genus Odontomachus. Studia Entomologica 19:67-171.

Brown WL Jnr. 1978. Contributions toward a reclassification of the Formicidae. Part VI. Ponerinae, tribe Ponerini, subtribe Odontomachiti. Section B. Genus Anochetus and bibliography. Studia Entomologica 20:549-638. 
Brown MJF. 1999. Semi-claustral founding and worker behaviour in gynes of Messor andrei. Insectes Sociaux 46:194-195. doi: 10.1007/s000400050133.

Brown MJF, Bonhoeffer S. 2003. On the evolution of claustral colony founding in ants. Evolutionary Ecology Research 5:305-313.

Crispo E. 2007. The Baldwin effect and genetic assimilation: revisiting two mechanism of evolutionary change mediated by phenotypic plasticity. Evolution 61:2469-2479. doi: 10.1111/j.1558-5646.2007.00203.x.

Cronin AL, Molet M, Doums C, Monnin T, Peeters C. 2013. Recurrent evolution of dependent colony foundation across eusocial insects. Annual Review of Entomology 58:37-55. doi: 10.1146/annurev-ento-120811-153643.

Dejean A. 2011. Prey capture behavior in an arboreal african ponerine ant. PLOS ONE 6:e19837. doi: 10.1371/ journal.pone.0019837.

Dejean A, Dejean A. 1998. How a ponerine ant acquired the most evolved mode of colony foundation. Insectes Soclaux 45:343-346. doi: 10.1007/s000400050093.

Dejean A, Delabie JHC, Corbara B, Azémar F, Groc S, Orivel J, Leponce M. 2012. The ecology and feeding habits of the arboreal trap-jawed ant Daceton armigerum. PLOS ONE 7:e37683. doi: 10.1371/journal.pone.0037683.

Dejean A, Fénéron R. 1996. Polymorphism and oligogyny in the ponerine ant Centromyrmex bequaerti (Formicidae: Ponerinae). Insectes Sociaux 43:87-99. doi: 10.1007/BF01253959.

Dejean A, Lachaud J-P. 1992. Growth-related changes in predation behavior in incipient colonies of the ponerine ant Ectatomma tuberculatum (Olivier). Insectes Sociaux 39:129-143. doi: 10.1007/BF01249289.

Delabie JHC, Reis YT. 2000. Sympatry and mating flight synchrony of three species of Cylindromyrmex (Hymenoptera: Formicidae) in southern Bahia, Brazil, and the importance of Malaise trap for rare ants inventory. Revista Brasileira de Entomologia 44:109-110.

Donoso D, Vieira JM, Wild A. 2006. Three new species of Leptanilloides Mann from Andean Ecuador (Formicidae: Leptanilloidinae). Zootaxa 1201:47-62.

Dudley R. 2002. The biomechanics of insects flight: form, function, evolution. Princeton: Princeton University Press.

Emlen DJ. 2001. Costs and the diversification of exaggerated animal structures. Science 291:1534-1536. doi: 10.1126/science.1056607.

Folgarait PJ. 1998. Ant biodiversity and its relationship to ecosystem functioning: a review. Biodiversity and Conservation 7:1221-1244. doi: 10.1023/A:1008891901953.

Gotwald WH Jnr, Cunningham-van Someren GR. 1976. Taxonomic and behavioral notes on the African ant, Aenictus eugenii Emery, with a description of the queen (Hymenoptera: Formicidae). JNY Entomol Soc 84:182-188.

Grimaldi D, Agosti D, Carpenter JM. 1997. New and rediscovered primitive ants (Hymenoptera: Formicidae) in Cretaceous amber from New Jersey, and their phylogenetic relationships. American Museum Novitates 3208:1-43. http://hdl.handle.net/2246/3615

Hartenstein V. 2006. The muscle pattern of Drosophila. In: Sink H, editor. Muscle development in Drosophila. New York: Springer Science+Business Media. p. 8-27.

Haskins CP. 1970. Researches in the biology and social behavior of primitive ants. In: Aronson L, Tobach E, Lehrman D, Rosenblatt JS, editors. Development and evolution of behavior. San Francisco: W. H. Freeman. p. 355-388.

Haskins CP, Haskins EF. 1950. Notes on the biology and social behavior of the archaic ponerine ants of the genera Myrmecia and Promyrmecia. Ann Entomol Soc Am 43:461-491.

Haskins CP, Haskins EF. 1951. Note on the method of colony foundation of the ponerine ant Amblyopone australis Erichson. The American Midland Naturalist 45:432-445. doi: 10.2307/2421735.

Hölldobler B, Carlin NF. 1985. Colony founding, queen dominance and oligogyny in the Australian meat ant Iridomyrmex purpureus. Behavioral Ecology and Sociobiology 18:45-58.

Hölldobler B, Wilson EO. 1978. The multiple recruitment system of the African weaver ant Oecophylla longinoda (Latreille) (Hymenoptera: Formicidae). Behavioral Ecology and Sociobiology 3:19-60. doi: 10.1007/BF00300045.

Hölldobler B, Wilson EO. 1990. The Ants. Cambridge, Massachusetts: Harvard University Press.

Ito F. 2010. Notes on the biology of the Oriental amblyoponine ant Myopopone castanea: Queen-worker dimorphism, worker polymorphism and larval hemolymph feeding by workers (Hymenoptera: Formicidae). Entomological Science 13:199-204. doi: 10.1111/j.1479-8298.2010.00384.x.

Ito F, Billen J. 1998. Larval hemolymph feeding and oophagy: behavior of queen and workers in the primitive ponerine ant Prionopelta kraepelini (Hymenoptera, Formicidae). Belgian Journal of Zoology 128:201-209.

Johnson RA. 2002. Semi-claustral colony founding in the seed-harvester ant Pogonomyrmex californicus: a comparative analysis of colony founding strategies. Oecologia 132:60-67. doi: 10.1007/s00442-002-0960-2.

Jones R, Davis W, Hung A, Vinson S. 1978. Insemination-induced histolysis of the flight musculature in fire ants (Solenopsis spp.): an ultrastructural study (1). The American Journal of Anatomy 151:603-610. doi: 10.1002/ aja.1001510411.

Kannowski PB. 1959. The flight activities and colony-founding behavior of bog ants in southeastern Michigan. Insectes Soclaux 6:115-162. doi: 10.1007/BF02225947.

Keller L, Passera L. 1989. Size and fat content of gynes in relation to the mode of colony founding in ants (Hymenoptera; Formicidae). Oecologia 80:236-240.

Keller RA. 2011. A phylogenetic analysis of ant morphology (Hymenoptera: Formicidae) with special reference to the poneromorph subfamilies. Bulletin of the American Museum of Natural History 355:1-90. doi: 10.1206/355.1. 
Keller RA, Peeters C, Beldade P. 2014. Data from: Evolution of thorax architecture in ant castes highlights trade-off between flight and ground behaviors. Dryad Digital Repository. doi: 10.5061/dryad.d62p2.

Kronauer DJC, Schöning C, Pedersen JS, Boomsma JJ, Gadau J. 2004. Extreme queen-mating frequency and colony fission in African army ants. Molecular Ecology 13:2381-2388. doi: 10.1111/j.1365-294X.2004.02262.x.

Lenoir A, Dejean A. 1994. Semi-claustral colony foundation in the formicine ants of the genus Polyrhachis (Hymenoptera: Formicidae). Insectes Soclaux 41:225-234. doi: 10.1007/BF01242293.

Lewis PO. 2001. A likelihood approach to estimating phylogeny from discrete morphological character data. Systematic Biology 50:913-925. doi: 10.1080/106351501753462876.

Lubertazzi D. 2012. The Biology and Natural History of Aphaenogaster rudis. Psyche (Cambridge) 2012:11.

Maddison WP. 1990. A method for testing the correlated evolution of two binary characters: are gains or losses concentrated on certain branches of a phylogenetic tree? Evolution 44:539-557. doi: 10.2307/2409434.

Maddison DR, Maddison WP. 2005. MacClade 4: analysis of phylogeny and character evolution. Version 4.08a ed. http://macclade.org

Maddison WP, Maddison DR. 2012. Mesquite: a modular system for evolutionary analysis. 2.0 ed. http://mesquiteproject.org

Masuko K. 1990. Behavior and Ecology of the Enigmatic Ant Leptanilla japonica Baroni Urbani (Hymenoptera: Formicidae: Leptanillinae). Insectes Soclaux 37:31-57. doi: 10.1007/BF02223813.

McQuilton P, St Pierre SE, Thurmond J, FlyBase Consortium. 2012. FlyBase 101 - the basics of navigating FlyBase. Nucleic Acids Research 40:D706-D714. doi: 10.1093/nar/gkr1030.

Mikó I, Friedrich F, Yoder MJ, Hines HM, Deitz LL, Bertone MA, Seltmann KC, Wallace MS, Deans AR. 2012. On dorsal prothoracic appendages in treehoppers (Hemiptera: Membracidae) and the nature of morphological evidence. PLOS ONE 7:e30137. doi: 10.1371/journal.pone.0030137.

Moczek AP, Emlen DJ. 2000. Male horn dimorphism in the scarab beetle, Onthophagus taurus: do alternative reproductive tactics favour alternative phenotypes? Animal behaviour 59:459-466. doi: 10.1006/anbe.1999.1342.

Moll K, Roces F, Federle W. 2010. Foraging grass-cutting ants (Atta vollenweideri) maintain stability by balancing their loads with controlled head movements. Journal of Comparative Physiology 196:417-480. doi: 10.1007/ s00359-010-0535-3.

Molet M, Fisher B, Ito F, Peeters C. 2009. Shift from independent to dependent colony foundation and evolution of 'multi-purpose' ergatoid queens in Mystrium ants (subfamily Amblyoponinae). Biological Jorunal of the Linnean Society 98:198-207. doi: 10.1111/j.1095-8312.2009.01257.x.

Moreau CS, Bell CD, Vila R, Archibald SB, Pierce NE. 2006. Phylogeny of the ants: diversification in the age of angiosperms. Science 312:101-104. doi: 10.1126/science.1124891.

Mori A, D'Ettorre P, Le Moli F. 1995. Host nest usurpation and colony foundation in the European amazon ant, Polyergus rufescens (Hymenoptera: Formicidae). Insectes Sociaux 42:279-286. doi: 10.1007/BF01240422.

Nijhout HF, Emlen DJ. 1998. Competition among body parts in the development and evolution of insect morphology. Proceedings of the National Academy of Sciences of the United States of America 95:3685-3689. doi: 10.1073/pnas.95.7.3685.

Nixon KC. 2002. WinClada. 1.00.08 ed. Ithaca, New York: Published by the author. http://www.cladistics.com

Oster GF, Wilson EO. 1978. Caste and ecology in the social insects. Princeton: Princeton University Press.

Paul J, Gronenberg W. 1999. Optimizing force and velocity: mandible muscle fibre attachments in ants. The Journal of Experimental Biology 202:797-808.

Peeters C. 1997. Morphologically "primitive" ants: comparative review of social characters, and the importance of queen-worker dimorphism. In: Choe J, Crespi B, editors. The evolution of social behavior in insects and Arachnids. Cambridge: Cambridge University Press. p. 372-391.

Peeters C. 2012. Convergent evolution of wingless reproductives across all subfamilies of ants, and sporadic loss of winged queens (Hymenoptera: Formicidae). Myrmecological News 16:75-91.

Prud'homme B, Minervino C, Hocine M, Cande JD, Aouane A, Dufour HD, Kassner VA, Gompel N. 2011. Body plan innovation in treehoppers through the evolution of an extra wing-like appendage. Nature 473:83-86. doi: 10.1038/nature09977.

R Development Core Team. 2008. R: a language and environment for statistical computing. 2.8.1 ed. R foundation for statistical computing. Vienna, Austria: http://www.r-project.org/

Rabeling C, Brown JM, Verhaagh M. 2008. Newly discovered sister lineage sheds light on early ant evolution. Proceedings of the National Academy of Sciences of the United States of America 105:14913-14917. doi: 10.1073/pnas.0806187105.

Robertson HG, Villet MH. 1989. Colony founding in the ant Carebara vidua: the dispelling of a myth. South African Journal Of Animal Science 85:121-122.

Robinson BW, Dukas R. 1999. The influence of phenotypic modifications on evolution: the Baldwin effect and modern perspectives. Oikos 85:582-589. doi: 10.2307/3546709.

Schneirla TC. 1949. Army-ant life and behavior under dry season conditions. 3. The course of reproduction and colony behavior. Bulletin of the American Museum of Natural History 94:1-82.

Simpson S, Sword G, Lo N. 2012. Polyphenism in insects. Current Biology 21:R738-R749. doi: 10.1016/j. cub.2011.06.006.

Simpson GG. 1953. The Baldwin effect. Evolution 7:110-117. doi: 10.2307/2405746.

Snodgrass RE. 1935. Principles of insect morphology. Ithaca, NY: Cornell University Press.

Snodgrass RE. 1956. Anatomy of the honey bee. Ithaca: Cornell University Press.

Stille M. 1996. Queen/worker thorax volume ratios and nest-founding strategies in ants. Oecologia 105:87-93. doi: 10.1007/BF00328795. 
Trager JC. 2013. Global revision of the dulotic ant genus Polyergus (Hymenoptera: Formicidae, Formicinae, Formicini). Zootaxa 3722:501-548. doi: 10.11646/zootaxa.3722.4.5.

Ward PS. 1981. Ecology and life history of the Rhytidoponera impressa group (Hymenoptera: Formicidae).

II. Colony origin, seasonal cycles, and reproduction. Psyche 88:109-126. doi: 10.1155/1981/39593.

Ward PS. 2007. Phylogeny, classification, and species-level taxonomy of ants (Hymenoptera: Formicidae). Zootaxa 1668:549-563.

Wcislo WT. 1989. Behavioral environments and evolutionary change. Annual Review of Ecology, Evolution, and Systematics 20:137-169. doi: 10.1146/annurev.es.20.110189.001033.

West-Eberhard MJ. 2003. Developmental plasticity and evolution. New York: Oxford University Press.

Wheeler DE, Buck NA. 1996. Depletion of reserves in ant queens during claustral colony founding. Insectes Soclaux 43:297-302. doi: 10.1007/BF01242930.

Wheeler DE. 1986. Developmental and physiological determinants of caste in social Hymenoptera: evolutionary implications. The American Naturalist 128:13-34. doi: 10.1086/284536.

Wilson EO, Eisner T, Wheeler GC, Wheeler J. 1956. Aneuretus simoni Emery, a major link in ant evolution.

Bulletin of the Museum of Comparative Zoology at Harvard College 115:81-99.

Wilson EO. 1971. The insect societies. Cambridge, Mass: Harvard University Press.

Yamauchi K, Furukawa T, Kinomura K, Takamine H, Tsuji K. 1991. Secondary polygyny by inbred wingless sexuals in the dolichoderine ant Technomyrmex albipes. Behavioral Ecology and Sociobiology 29:313-319. doi: 10.1007/BF00165955.

Yoshizawa K. 2011. The treehopper's helmet is not homologous with wings (Hemiptera: Membracidae). Systematic Entomology 37:2-6. doi: 10.1111/j.1365-3113.2011.00606.x. 